\title{
Article \\ A Finite Element Study on Compressive Resistance Degradation of Square and Circular Steel Braces under Axial Cyclic Loading
}

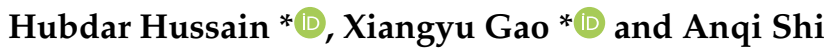 \\ College of Architecture and Civil Engineering, Beijing University of Technology, Beijing 100124, China; \\ ly101019@163.com \\ * Correspondence: hubdarkhan@gmail.com (H.H.); gaoxy@bjut.edu.cn (X.G.)
}

Citation: Hussain, H.; Gao, X.; Shi, A

A Finite Element Study on

Compressive Resistance Degradation of Square and Circular Steel Braces under Axial Cyclic Loading. Appl. Sci. 2021, 11, 6094. https://doi.org/ 10.3390/app11136094

Academic Editors: Angelo Luongo and Daniele Zulli

Received: 24 May 2021

Accepted: 27 June 2021

Published: 30 June 2021

Publisher's Note: MDPI stays neutral with regard to jurisdictional claims in published maps and institutional affiliations.

Copyright: (c) 2021 by the authors. Licensee MDPI, Basel, Switzerland This article is an open access article distributed under the terms and conditions of the Creative Commons Attribution (CC BY) license (https:// creativecommons.org/licenses/by/ $4.0 /)$.
Abstract: In this study, detailed finite element analysis was conducted to examine the seismic performance of square and circular hollow steel braces under axial cyclic loading. Finite element models of braces were constructed using ABAQUS finite element analysis (FEA) software and validated with experimental results from previous papers to expand the specimen's matrix. The influences of cross-section shape, slenderness ratio, and width/diameter-to-thickness ratio on hysteretic behavior and compressive-tensile strength degradation were studied. Simulation results of parametric studies show that both square and circular hollow braces have a better cyclic performance with smaller slenderness and width/diameter-to-thickness ratios, and their compressive-tensile resistances ratio significantly decreases from cycle to cycle after the occurrence of the global buckling of braces.

Keywords: seismic performance; braces; axial cyclic loading; finite element models; compressivetensile strength degradation

\section{Introduction}

Concentric bracing frames (CBFs) are earthquake-resistant structures that are easy to design, economical, and efficient at resisting lateral forces, and limit the deformation in building structures that face wind and earthquake loading. These frames provide sufficient stiffness and lateral strength to structural systems to fulfill the requirements of serviceability and operability under seismic events. Conventional diagonal braces are considered the most influential components of this system because they provide adequate displacement ductility and have good energy dissipation potential. These braces undergo large inelastic axial deformations to protect the primary frame members of the gravity system and prevent collapsing under earthquake loadings. Thus, braces act as a fuse and undergo repetitive buckling under compression loads and yielding under tension forces. However, the steel braces used in special concentric braced frames (SCBFs) possess better tensile capacity than buckling capacity, which results in an unbalanced performance under seismic loading, and notably weakens the efficacy of frames in dissipating the inserted seismic energy [1]. The asymmetric behavior of steel braces further decays their load-bearing capacity and compressive strength due to the creation of a plastic hinge at the middle of the brace after the occurrence of buckling. The buckling and unequal response of steel braces can lead to the deterioration of the seismic endurance of the braced structures, and may significantly increase the inelastic torsion of the structure [2]. Therefore, careful design of SCBFs is necessary, and selected braces should be capable of providing sufficient strength and energy absorption capacity under possible cyclic loading.

Numerous studies [3-10] have been conducted with the aim of providing a better framework to select braced frame sections and their configurations, improved gusset plate connections, and the design of columns and beams to achieve enhanced seismic performance of SCBFs. Hollow square and circular sections are mostly used as braces in special concentric braced frames (SCBFs), and significant experimental and numerical research has been undertaken to examine the hysteretic load-displacement response of these brace 
sections [11-16]. These previous studies have provided significant data to understand the key parameters of steel braces under cyclic loadings, such as energy dissipation capacity, ductility, lateral deflection at mid-span, and post-buckling compressive resistance. Contrary to other important parameters, asymmetric compressive-tensile resistance ratios, which influence the seismic performance of braced structures, have not been previously investigated. Thus, examination of these parameters is required.

Hollow square and circular braces are preferred due to their easier connection specifications and applicability to a wide range of sections; the corresponding dimensions (width, diameter, and thickness) of both square and circular specimens are shown in Figure 1. To examine seismic performance, a comprehensive finite element study was conducted on a broad range of slenderness values and width/diameter-to-thickness ratios of square and circular hollow braces using ABAQUS v.6.10 finite element (FE) software [17]. First, finite element models of braces were developed based on experimental tests conducted by $[1,15]$ to replicate the axial load hysteretic response for parametric study of braces under axial cyclic loading. Then, following the FE models validated against experimental results, a series of parametric studies were carried out on a wide array of specimens to assess hysteretic performance, energy dissipation capability, and asymmetric compressive-tensile resistance.

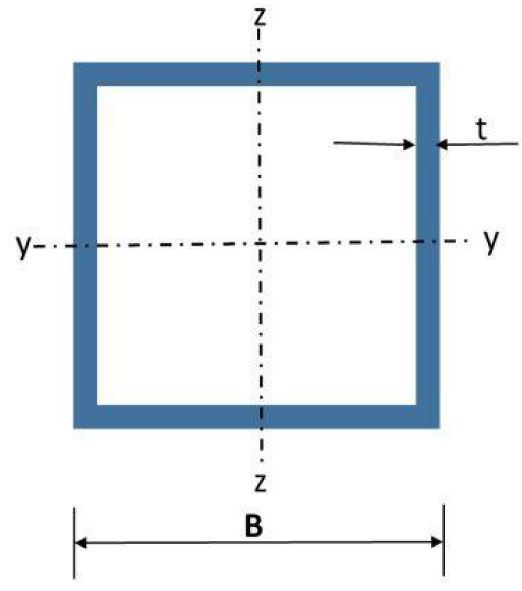

(a)

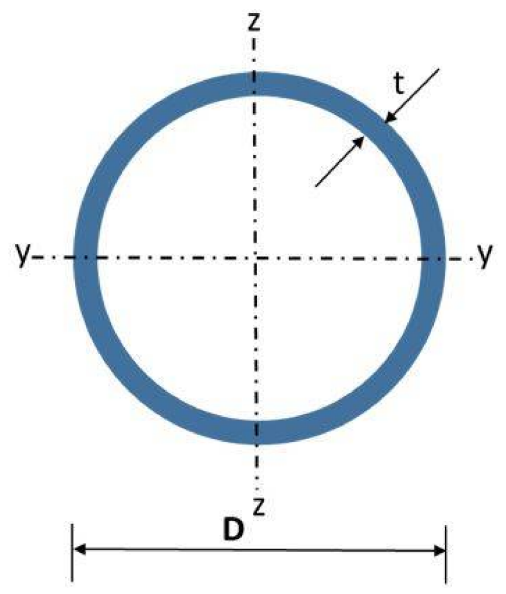

(b)

Figure 1. Corresponding dimensions of (a) Square shaped specimens (b) Circular Shaped specimens.

\section{Numerical Study}

The three-dimensional continuum-based finite element models of hollow square and circular braces were developed using ABAQUS general-purpose software. All of the developed brace models included the inelastic behavior of brace components and stiffen plate connection effects. A wide-ranging series of FE models with various slenderness ratios and width/diameter-to-thickness ratios of brace sections were developed in this numerical study, and details about the development of the FE models, the loading protocol, validation, and analysis of results are presented in the subsequent sections.

\subsection{Element Type and Meshing}

To save computational time and enable inelastic behavior of the braces, a threedimensional four-node doubly curved quadrilateral general-purpose reduced integrated and structured shell element (S4R) was employed throughout this finite element study. This element type, which included five integration points and six degrees of freedom per node (three translational and three rotational), allows adequate nonlinear stress distribution and transverse shear deformation for thick shell or thin shell appliances, and has been effectively utilized in previous studies for numerical modeling of hollow steel bracing and compression members to accurately predict compressive resistance and failure modes. All numerical braces were partitioned into three portions of different mesh sizes along the 
longitudinal direction, as shown in Figure 2. More refined mesh having a size of about half the section thickness was employed in mid-span and end regions, where high inelastic deformations were expected under cyclic loading. In both directions from the mid-span, the fine element mesh was extended about 1.5 times the width/diameter of the sections. A coarser mesh with a size of about twice the hollow section thickness was adopted in the remaining parts of the simulated braces for computational efficiency.

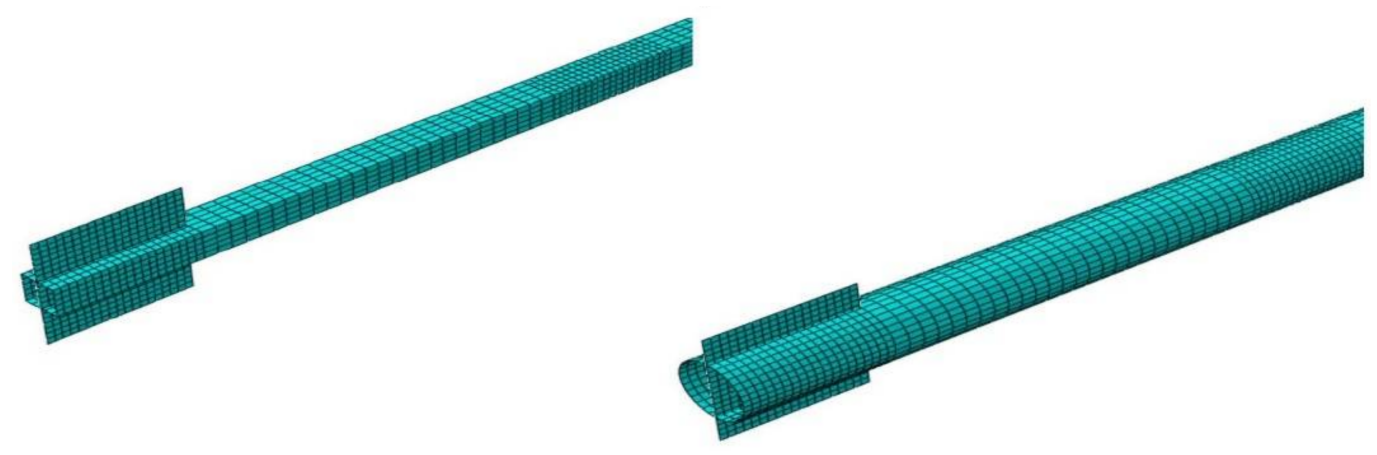

Figure 2. Mesh pattern of braces.

\subsection{Loading Protocol and End Conditions}

Each earthquake has unique characteristics, and loading histories cannot represent real earthquake movements. Different testing protocols have been developed globally and adopted to deduce the seismic performance of entire structures, sub-structures, and structural members under cyclic loading. The loading protocol recognized by the European Convention for Constructional Steelwork (ECCS) [18] is considered to be appropriate for the assessment of cyclic behavior of steel tubular sections and has been employed in an ongoing study to enable comparison of results with [1,15]. The loading sequence comprised one cycle at a displacement amplitude of $0.25 \Delta y, 0.5 \Delta y, 0.75 \Delta y$, and $1.0 \Delta y$, followed by three cycles of $2 \Delta y, 4 \Delta y, 6 \Delta y, 8 \Delta y$, etc., as shown in Figure 3. The standardized axial cyclic displacement $\Delta \mathrm{a} / \Delta \mathrm{y}$ is the governing parameter of the loading protocol, where $\Delta \mathrm{a}$ is the applied displacement at the loaded end and $\Delta y$ is the tensile yield displacement obtained by the product of yield strain and member length $[1,15]$. The loading path was symmetric in tension and compression, and specimens were first loaded in compression and then in tension. A fixed-fixed boundary condition was applied and, in finite element modeling, the end sections were coupled to a reference point at each side and all degrees of freedom were restrained, with the exception that longitudinal translation was allowed at the loaded end for the application of axial displacement.

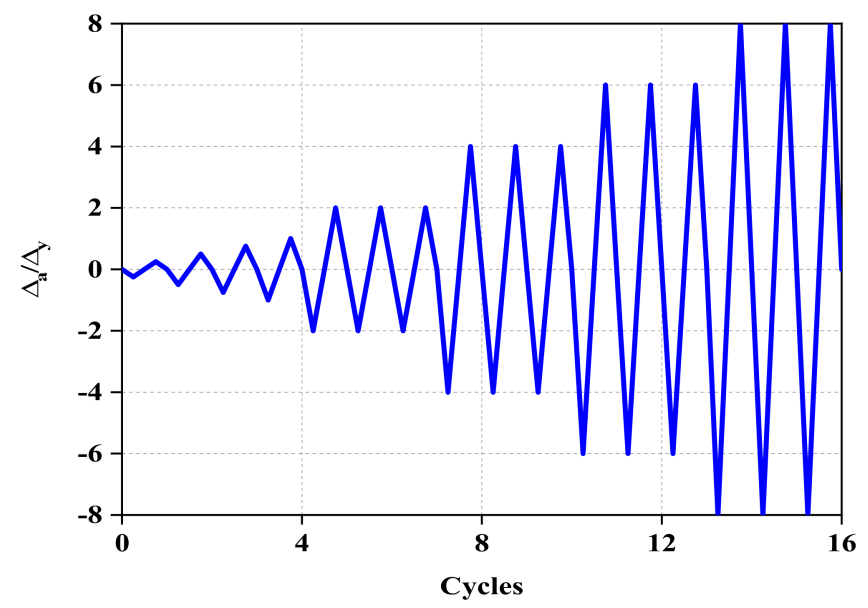

Figure 3. ECCS [18] loading protocol ( $\Delta \mathrm{a} / \Delta \mathrm{y}=$ ratio of applied displacement to the yield displacement). 


\subsection{Material Modelling}

To consider the inelastic behavior of materials under cyclic loading, a nonlinear combined isotropic/kinematic hardening material model was adopted in this paper for finite element analysis. This model was developed based on work by Lemaitre and Caboche [19], and can be used to represent cyclic hardening and the kinematic shift of the yield surface in a multi-dimensional stress space, as shown in Figure 4. The non-linear kinematic hardening element retains the same size and shape of the yield surface, but changes with additional stress cycles. The kinematic hardening comprised two terms: a linear kinematic phase followed by the Ziegler hardening law, and a back stress interval that defines the non-linearity. The mathematical expression of non-linear kinematic hardening is as follows:

$$
\dot{\alpha}_{k}=C_{k} \frac{1}{\sigma^{0}}(\sigma-\alpha) \frac{\dot{-}}{\epsilon}-\gamma_{k} \alpha_{k} \frac{\dot{x}}{\epsilon} p
$$

where $\sigma^{0}$ is the preliminary yield stress, $\dot{\bar{\epsilon}} p$ denotes the corresponding plastics strain level, and $C_{k}$ and $\gamma_{k}$ are material constants. $C_{k}$ signifies the first kinematic hardening moduli and $\gamma_{k}$ governs the kinematic hardening moduli diminution rate with respect to the accelerating plastic disfigurement. The total back-stress $(\alpha)$ is calculated using the given formula:

$$
\alpha=\sum_{k=1}^{N} \alpha_{k}
$$

where $N$ represents the total number of back-stresses. The isotropic hardening element, which tends to maintain an identical shape of the yield surface and allows the size to expand with increasing stress, can be illustrated as a direct function of the corresponding plastic strain, $\epsilon^{p}$, which jointly reflects the softening and hardening of the material. This can be computed as follows:

$$
\sigma^{0}=\sigma^{0}\left(\bar{\epsilon}^{p}\right)=\left.\sigma\right|_{0}+Q_{\infty}\left(1-e^{-b \bar{\epsilon}^{p}}\right)
$$

where $Q_{\infty}$ and $b$ are material parameters, and $\left.\sigma\right|_{0}$ signifies the yield stress at zero strain. $Q_{\infty}$ represents the extreme variation in yield surface size, whereas $b$ indicates the rate of variation for the yield surface during the development of plastic straining.

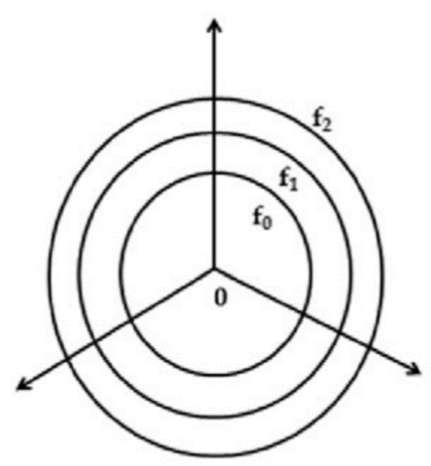

a)

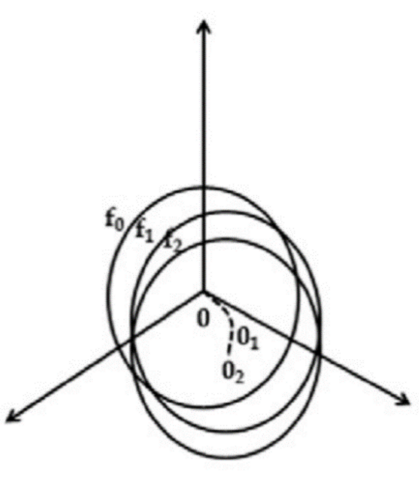

b)

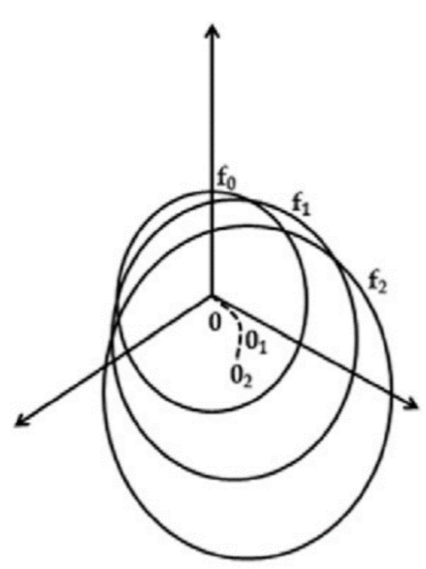

c)

Figure 4. (a) Rule of isotropic hardening; (b) rule of kinematic hardening; (c) joint hardening.

The nonlinear parameters of hot-rolled carbon steel required for simulation of the aforementioned model were taken from [20], as summarized in Table 1. These were applied to all numerical models throughout the study irrespective of brace size, geometry, and displacement levels of loading. All tubular structural members contain initial geometric imperfections, which mainly originate during the manufacturing process and have a significant influence on the endurance of the members. In this numerical study, both 
global and local initial geometric imperfections were included in the simulation models to consider their impact. Initially, an elastic eigenvalue buckling analysis was conducted to obtain the lowest buckling mode shapes shown in Figures 5 and 6 . Then, respective amplitudes (L/1500, $\mathrm{t} / 100)$ of global and local imperfections, as suggested in previous studies [15], were applied in non-linear analysis corresponding to the eigenvector of the first mode shape.

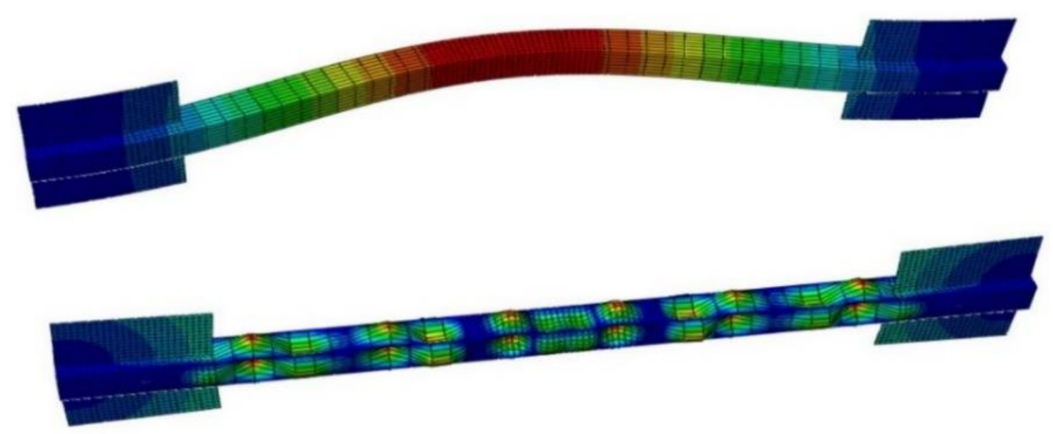

Figure 5. Effects of geometric imperfections in square braces: global buckling mode (amplitude $=\mathrm{L} / 1500$ ); local buckling mode (amplitude $=\mathrm{t} / 100$ ).
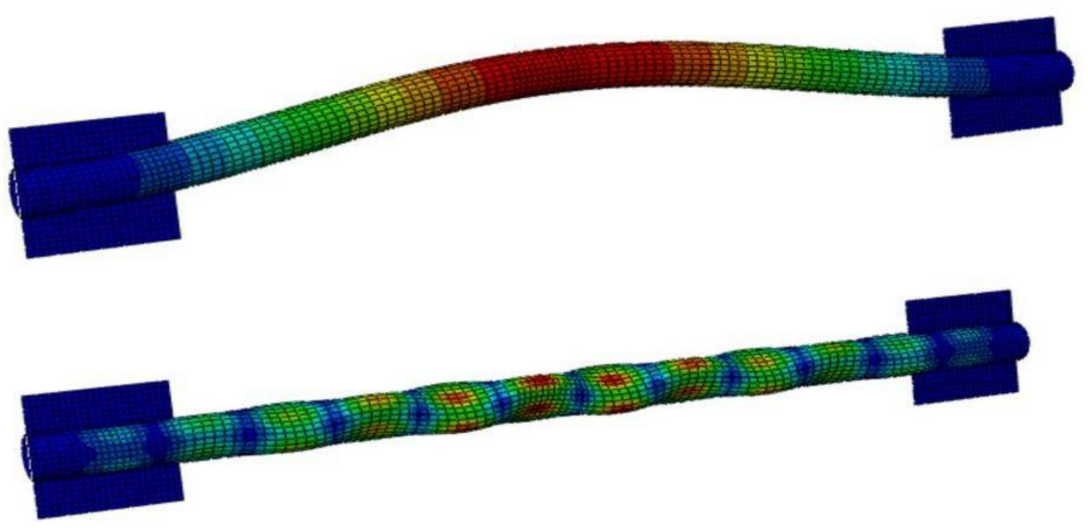

Figure 6. Effects of geometric imperfections in circular braces: global buckling mode (amplitude $=\mathrm{L} / 1500$ ); local buckling mode (amplitude $=\mathrm{t} / 100$ ).

Table 1. Nonlinear parameters of hot-rolled carbon steel [20].

\begin{tabular}{cc} 
Type of Material & Hot-Rolled Carbon Steel \\
\hline Young's Modulus, $E\left(\mathrm{kN} / \mathrm{mm}^{2}\right)$ & 215 \\
Density, $\rho\left(\mathrm{kg} / \mathrm{m}^{3}\right)$ & 7850 \\
Poison's ratio, $v$ & 0.3 \\
Minimum specified tensile yield strength, $f_{y}\left(\mathrm{~N} / \mathrm{mm}^{2}\right)$ & 355 \\
Measured tensile strength $/ 0.2 \%$ proof stress, $f_{y}$, meas $\left(\mathrm{N} / \mathrm{mm}^{2}\right)$ & 465 \\
Ultimate tensile strength, $f_{u}\left(\mathrm{~N} / \mathrm{mm}^{2}\right)$ & 540 \\
Yield stress at zero equivalent plastic strain, $\left.\sigma\right|_{\mathrm{o}}\left(\mathrm{N} / \mathrm{mm}^{2}\right)$ & 465 \\
Kinematic hardening constant, $C_{k i n}\left(\mathrm{~N} / \mathrm{mm}^{2}\right)$ & 23,554 \\
Kinematic hardening rate, $\gamma$ & 139 \\
Isotropic hardening constant, $Q_{\infty}\left(\mathrm{N} / \mathrm{mm}^{2}\right)$ & 55 \\
Isotropic hardening rate, $b_{i s o}$ & 2.38 \\
\hline
\end{tabular}

\subsection{Validation of FE Models}

The adopted FE modelling approach of this study led to an agreeable accordance and the developed FE models were validated against previous experimental studies conducted by $[1,15]$. The same boundary conditions, loading protocol, braces geometries, and material 
properties were applied as in the experimental test specimens. The comparison between FE predicted hysteresis axial force-displacement responses and test results are displayed in Figure 7. These results demonstrate that the material modeling approach adopted in the current FE study can precisely replicate the key features of experimental hysteresis behavior of the steel braces. Therefore, the FE modeling approach adopted in the current study is able to simulate the hysteretic behavior well for a large number of square and circular braces under cyclic loading. The dissimilarities between the experimental and FE results shown in Figure 7 could be due to the initial elastic stiffness through the elastic unloading, which resulted in a steeper drop in the unloading curves than those of the test specimens.

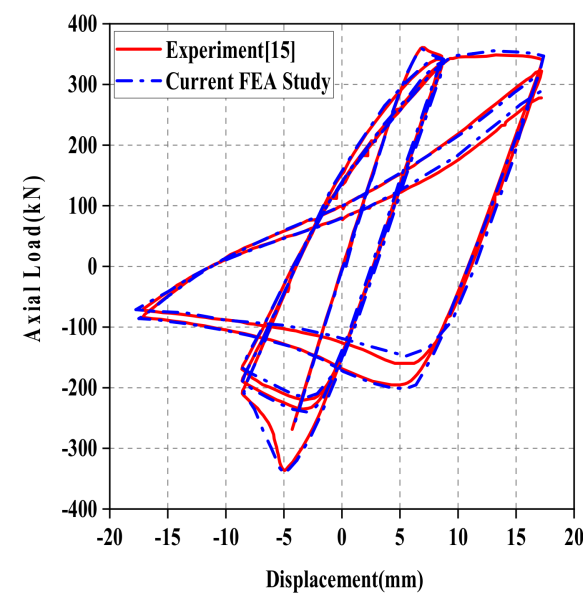

(a)

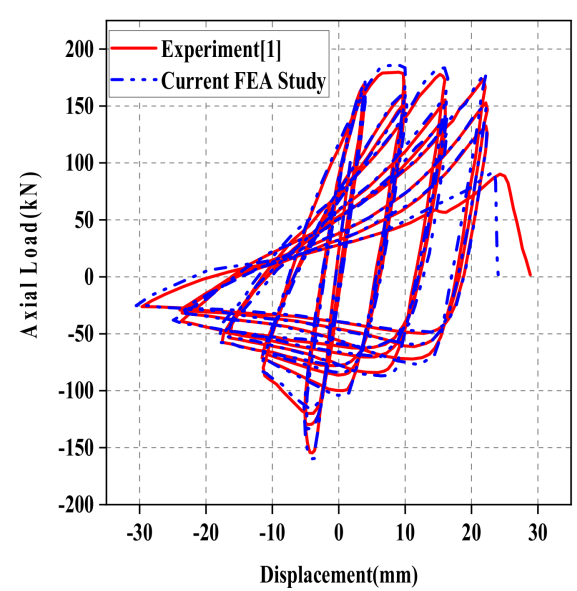

(b)

Figure 7. Validation of experiment results and current finite element study (a) $60 \times 60 \times 3 \times$ 2050 square specimen; (b) $48.3 \times 3.2 \times 1500$ circular specimen under axial cyclic loading with fixed-fixed end conditions.

\subsection{Design of Braces for Parametric Study}

Continuing with the validated FE modeling approach, a series of square and circular brace dimensions were chosen to investigate their cyclic behavior and compressive resistance. In this study, the slenderness ratio $(\lambda=\mathrm{L} / \mathrm{r})$ is defined as the length of the specimen to the radius of gyration, and its range for the selected braces was maintained at 50-150 with an increment of 10, as per the requirements of ANSI/AISC 341-16 and GB 50011-2010 [21,22]. Correspondingly, the B/t and D/t were altered from 8 to 20 with an increment of 4 , and the width of the square braces for calculation was taken as B-3t. To ensure this study was consistent with realistic engineering applications, an identical length of all braces equal to $3500 \mathrm{~mm}$ was adopted for buildings with the same floor height. The values of width/diameter-to-thickness ratio were varied to satisfy the required slenderness ratio, as presented in Table 2 .

Table 2. Geometric details of braces $(B=$ width; $t=$ thickness; $B / t=$ width-to-thickness ratio; $\mathrm{D}=$ diameter; $\mathrm{D} / \mathrm{t}=$ diameter-to-thickness ratio; $\mathrm{SR}=$ slenderness ratio).

\begin{tabular}{cccccccc}
\hline $\mathbf{B}(\mathbf{m m})$ & $\mathbf{t}(\mathbf{m m})$ & $\mathbf{B} / \mathbf{t}$ & $\mathbf{S R}$ & $\mathbf{D}(\mathbf{m m})$ & $\mathbf{t} \mathbf{( m m})$ & $\mathbf{D} / \mathbf{t}$ & $\mathbf{S R}$ \\
\hline 187 & 16.5 & 8 & 50 & 227 & 29 & 8 & 50 \\
182 & 12.5 & 12 & & 220 & 19.2 & 12 & \\
179 & 9.5 & 16 & & 215 & 13.9 & 16 & \\
177 & 7.7 & 20 & & 212 & 11 & 20 & \\
161 & 15 & 8 & 60 & 191 & 25.5 & 8 & 60 \\
157 & 11 & 12 & & 182 & 16 & 12 & \\
154 & 8.5 & 16 & & 178 & 11.3 & 16 & \\
152 & 6.8 & 20 & & 176 & 9 & 20 & \\
\hline
\end{tabular}


Table 2. Cont.

\begin{tabular}{|c|c|c|c|c|c|c|c|}
\hline$B(\mathrm{~mm})$ & $\mathrm{t}(\mathrm{mm})$ & $B / t$ & SR & $\mathrm{D}(\mathrm{mm})$ & $\mathrm{t}(\mathrm{mm})$ & $D / t$ & SR \\
\hline 135 & 12 & 8 & 70 & 164.5 & 23 & 8 & 70 \\
\hline 133 & 9 & 12 & & 156 & 14 & 12 & \\
\hline 128 & 6.5 & 16 & & 152 & 9.8 & 16 & \\
\hline 127 & 5.5 & 20 & & 148 & 7.6 & 20 & \\
\hline 119 & 10.8 & 8 & 80 & 143 & 19.2 & 8 & 80 \\
\hline 115 & 7.6 & 12 & & 136 & 12.3 & 12 & \\
\hline 113.5 & 6 & 16 & & 133 & 8.5 & 16 & \\
\hline 111 & 4.75 & 20 & & 131 & 6.8 & 20 & \\
\hline 105.5 & 9.5 & 8 & 90 & 127 & 17.8 & 8 & 90 \\
\hline 102 & 6.8 & 12 & & 121.5 & 11 & 12 & \\
\hline 100 & 5.2 & 16 & & 118 & 7.8 & 16 & \\
\hline 99 & 4.2 & 20 & & 115.7 & 6 & 20 & \\
\hline 95 & 8.6 & 8 & 100 & 114.5 & 16 & 8 & 100 \\
\hline 92.3 & 6.16 & 12 & & 109 & 9.7 & 12 & \\
\hline 90 & 4.6 & 16 & & 106 & 6.8 & 16 & \\
\hline 89 & 3.8 & 20 & & 105.2 & 5.5 & 20 & \\
\hline 86.5 & 8 & 8 & 110 & 103 & 14 & 8 & 110 \\
\hline 84 & 5.6 & 12 & & 98.6 & 8.5 & 12 & \\
\hline 82.8 & 4.4 & 16 & & 96.5 & 6.3 & 16 & \\
\hline 81.2 & 3.5 & 20 & & 95.5 & 5 & 20 & \\
\hline 79.6 & 7.3 & 8 & 120 & 94 & 12.5 & 8 & 120 \\
\hline 76.5 & 5.2 & 12 & & 90.1 & 7.8 & 12 & \\
\hline 76 & 4 & 16 & & 88.8 & 5.9 & 16 & \\
\hline 74.9 & 3.2 & 20 & & 87.1 & 4.5 & 20 & \\
\hline 73 & 6.8 & 8 & 130 & 86.6 & 11.6 & 8 & 130 \\
\hline 71 & 4.8 & 12 & & 83 & 7.4 & 12 & \\
\hline 70 & 3.7 & 16 & & 81 & 5.2 & 16 & \\
\hline 69 & 3.1 & 20 & & 80.7 & 4.2 & 20 & \\
\hline 68 & 6.3 & 8 & 140 & 80 & 10.3 & 8 & 140 \\
\hline 66.2 & 4.5 & 12 & & 77.5 & 6.9 & 12 & \\
\hline 65 & 3.4 & 16 & & 76 & 5 & 16 & \\
\hline 64.1 & 2.8 & 20 & & 74 & 3.8 & 20 & \\
\hline 63.1 & 5.5 & 8 & 150 & 73.5 & 8.9 & 8 & 150 \\
\hline 62 & 4.2 & 12 & & 72 & 6.4 & 12 & \\
\hline 61 & 3.3 & 16 & & 70.6 & 4.6 & 16 & \\
\hline 60 & 2.6 & 20 & & 69 & 3.5 & 20 & \\
\hline
\end{tabular}

\section{Results}

\subsection{Axial Load-Displacement Hysteretic Response}

Axial load vs. displacement curves of the square and circular braces for distinctive values of slenderness ratio and width/diameter-to-thickness ratios under the axial cyclic loadings are shown in Figures 8 and 9. Specimens with the lower values of slenderness ratio and width-to-thickness ratio have bigger circumscribed areas of axial load-displacement hysteresis loops per cycle. For a specified value of slenderness ratio $\lambda$, such as 50 , the total axial resistance of specimens in terms of tension and compression reduced with the increment in width-to-thickness ratio. For instance, as the value of the $\mathrm{B} / \mathrm{t}$ ratio increased from 8 to 20, the maximum tensile and compressive resistances reduced gradually for all slenderness ratios. In addition, comparing the hysteretic response at different values of width/diameter-to-thickness ratios, the axial resistances of braces reduced as the slenderness ratio $\lambda$ increased. During the first and subsequent cycles for the same amplitude loading, the tensile and compressive resistances decreased gradually and the hysteresis loops coincided at lower slenderness ratios. In contrast, at higher slenderness ratios, the gap between cycles increased, which was apparent for slenderness ratios exceeding 90. 

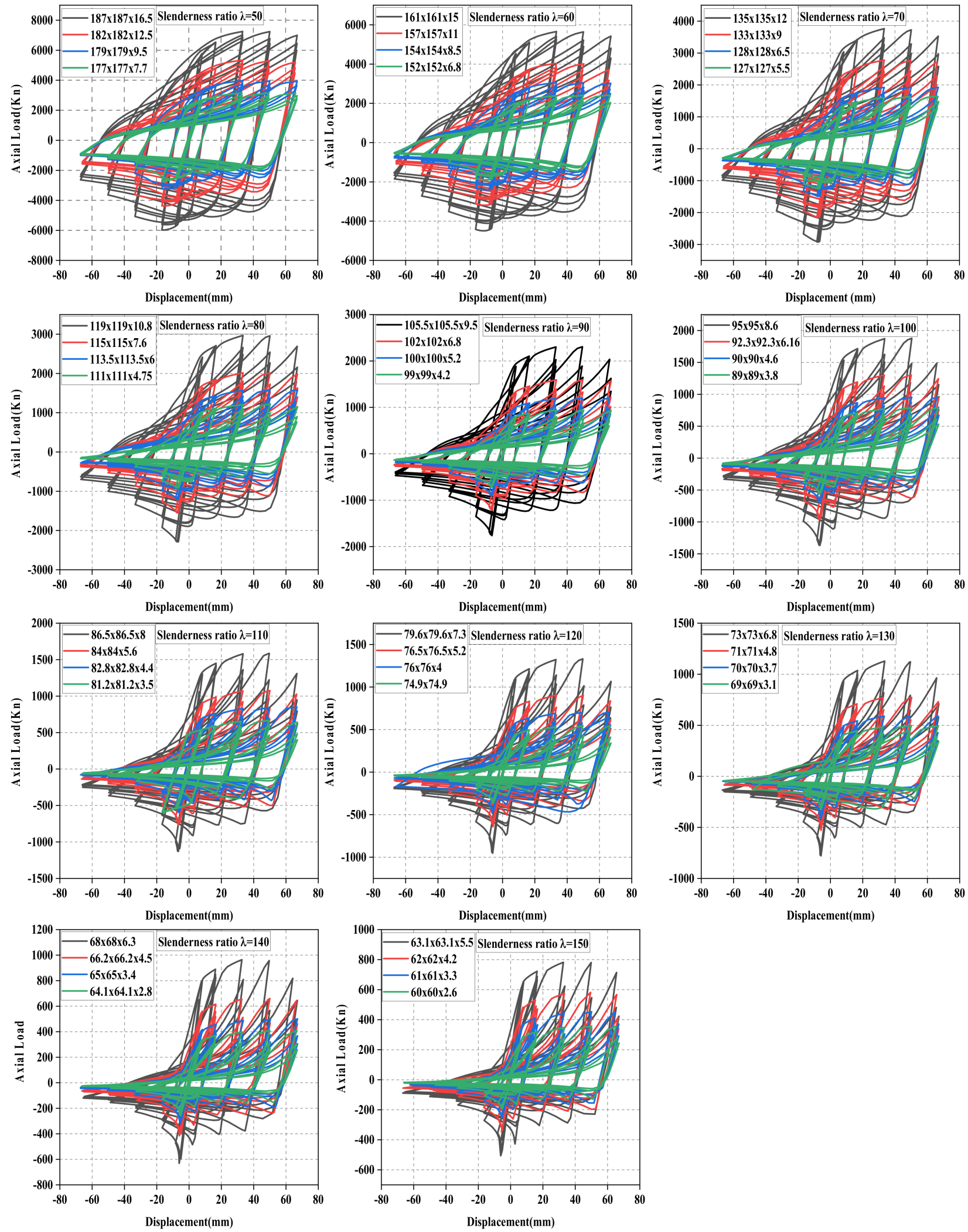

Figure 8. Cyclic response of hot-rolled carbon steel square braces under axial cyclic loading with fixed-fixed end conditions. 

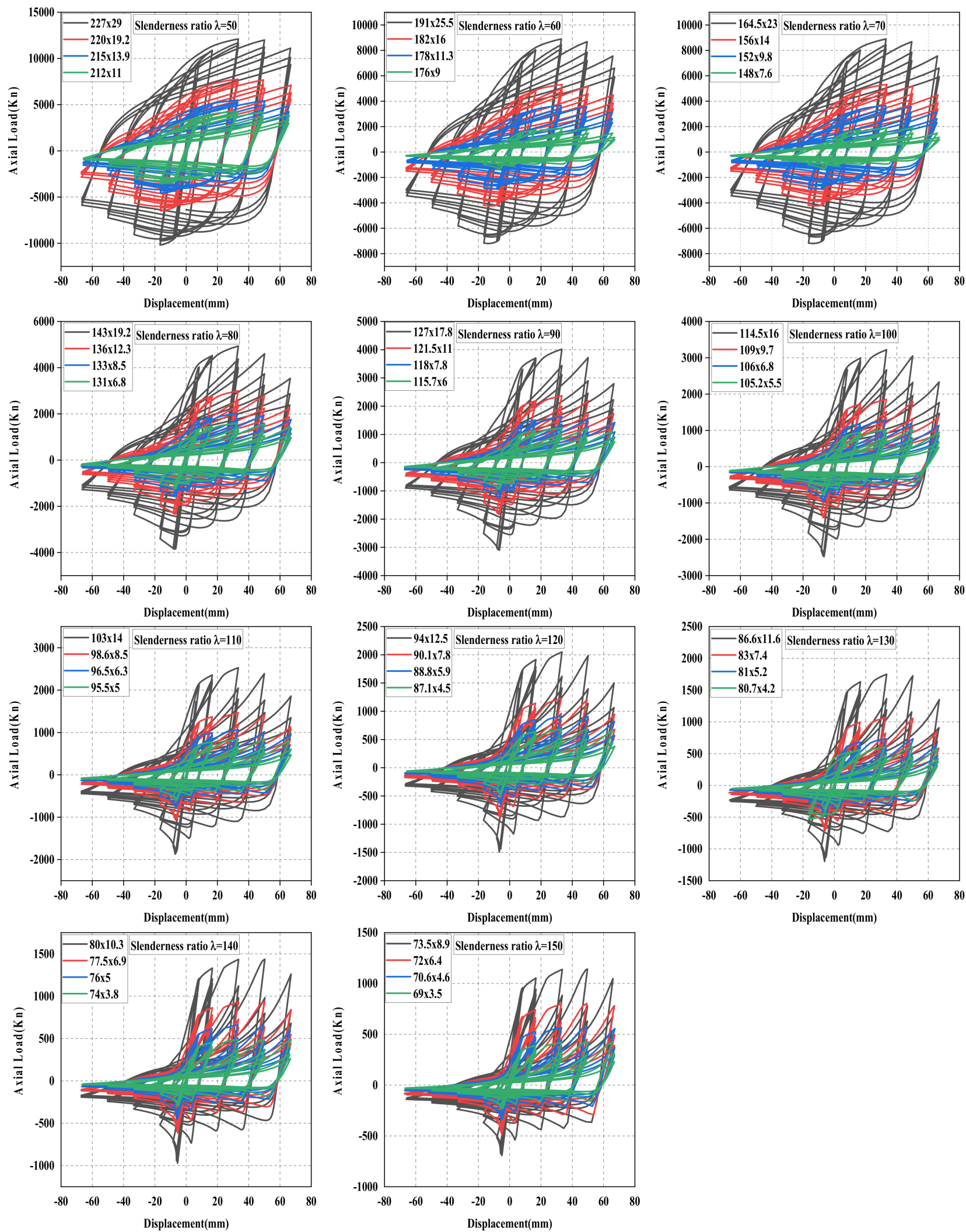

Figure 9. Cyclic response of hot-rolled carbon steel circular braces under axial cyclic loading with fixed-fixed end conditions.

\subsection{Compressive/Tensile Strength Ratios Per Load Cycle}

It has been previously noted that the hysteretic performance of steel braces relies on the parameters of energy dissipation capacity, ductility, lateral deflection at mid-span, 
and post-buckling compressive resistance. To fulfill the torsion requirements of structures, stable compressive-tensile resistances of the bracing members are necessary to ensure the adequate performance of the SCBFs during strong earthquakes. The main goal of this numerical study was to investigate the compressive-tensile strength ratios for square and circular braces, and their curves are presented in Figures 10 and 11. As shown by the results, both square and circular braces exhibited similar degradation of compressive-tensile strengths with the applied loading. During the initial cycles with small displacement amplitudes, the compressive-tensile resistances remained stable until the global buckling of braces; subsequently, the compressive-tensile strength ratio gradually decreased from cycle to cycle. The compressive-tensile strengths ratio declined rapidly under large amplitude loading.

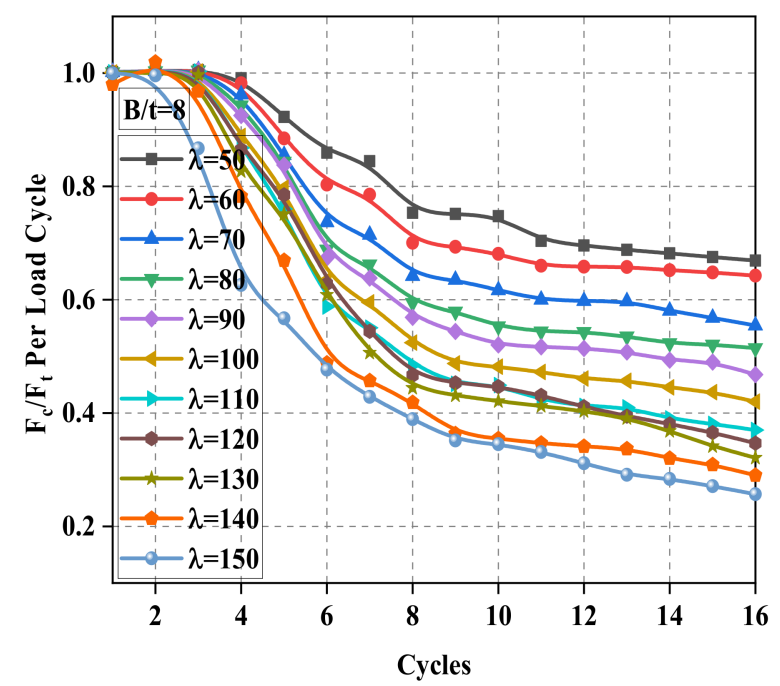

(a)

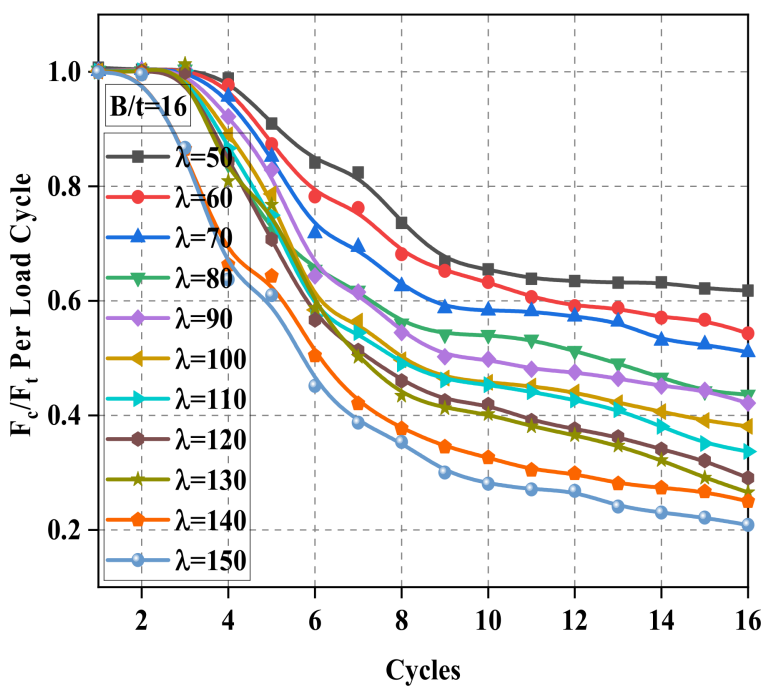

(c)

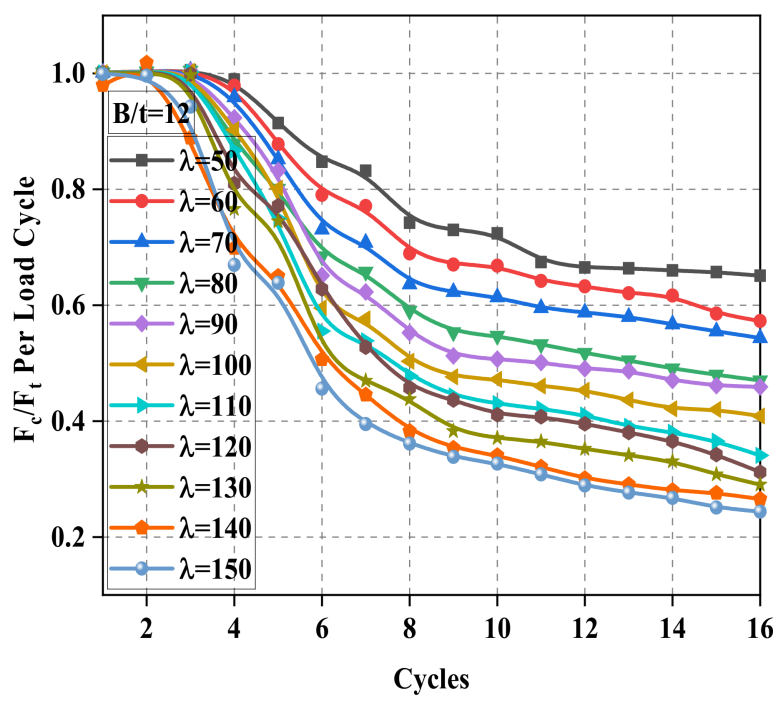

(b)

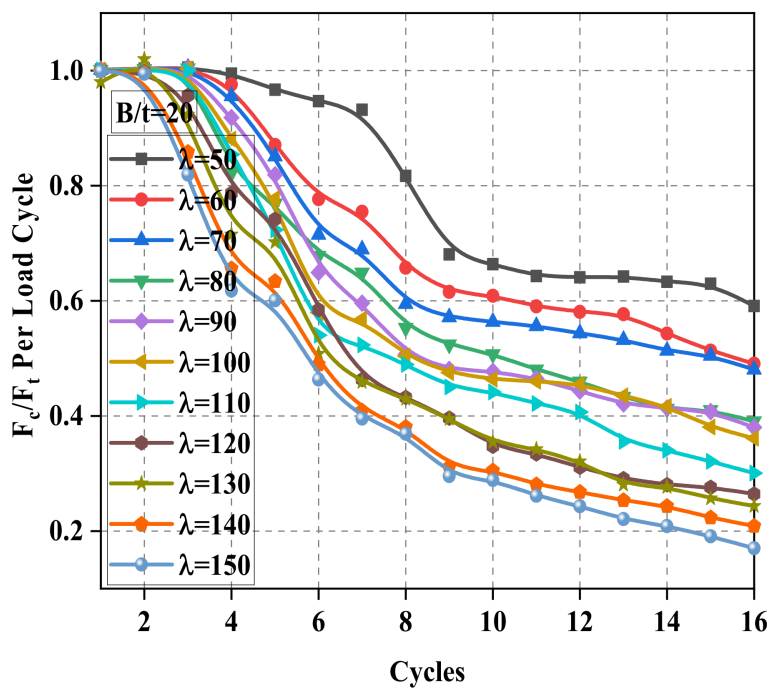

(d)

Figure 10. Compressive/tensile resistance ratios of square braces for slenderness ratios $(\lambda=50-150)$ : (a) width-to-thickness ratio $=8 ;(\mathbf{b})$ width-to-thickness ratio $=12 ;(\mathbf{c})$ width-to-thickness ratio $=16 ;(\mathbf{d})$ width-to-thickness ratio $=20$. 


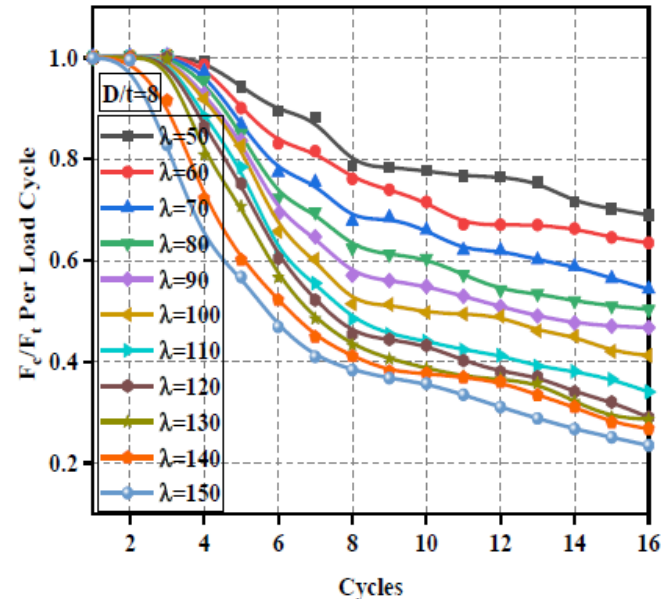

(a)

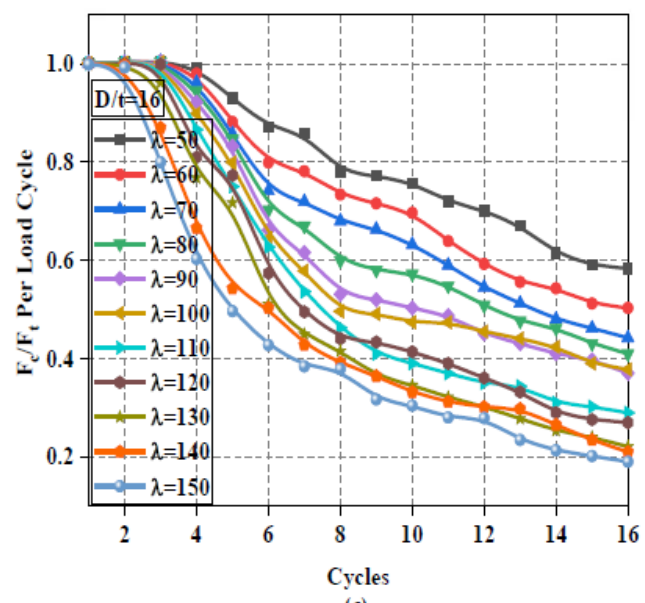

(c)

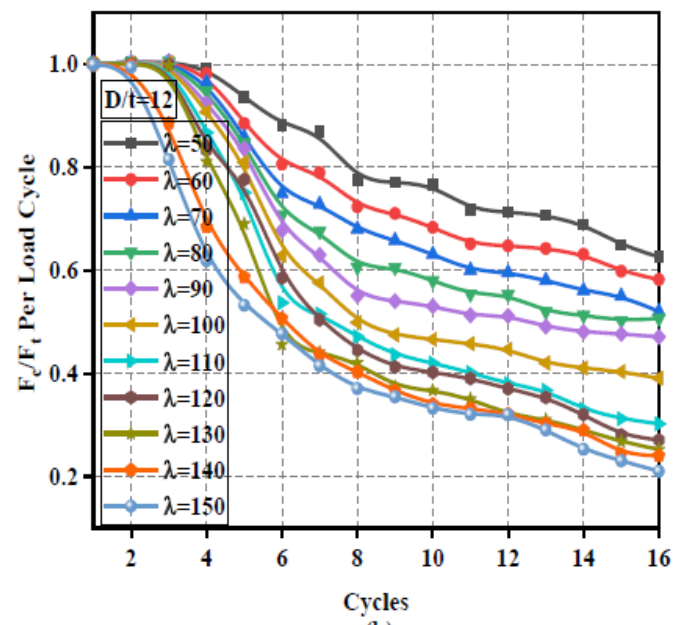

(b)

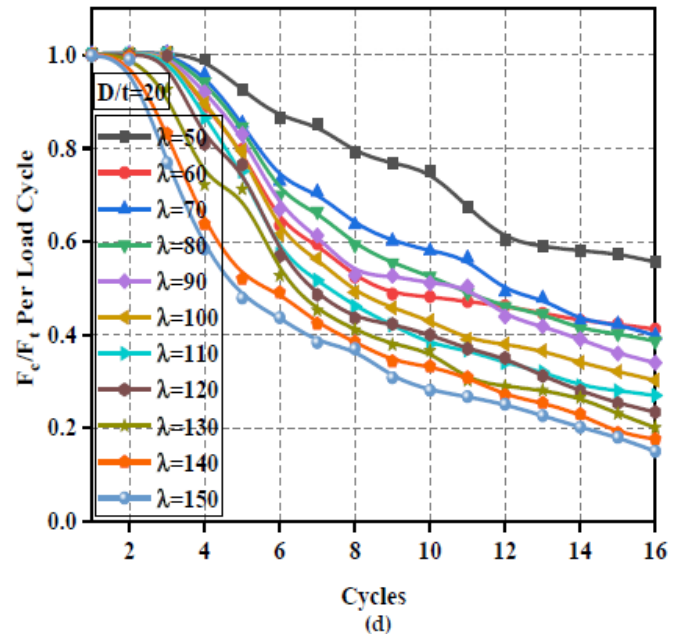

Figure 11. Compressive/tensile resistance ratios of circular braces for slenderness ratios $(\lambda=50-150)$ : (a) diameter-to-thickness ratio $=8 ;(\mathbf{b})$ diameter-to-thickness ratio $=12 ;(\mathbf{c})$ diameter-to-thickness ratio $=16 ;(\mathbf{d})$ diameter-to-thickness ratio $=20$.

\subsection{Cumulative Energy Dissipation of Braces}

The energy dissipation capacity of braces has a substantial influence on the seismic performance of SCBFs and can be quantitatively computed from the area bounded in axial load-displacement curves. During the initial cycles of elastic loading, the specimens normally display relatively symmetrical axial load-displacement responses, with insignificant hysteresis loops and dissipate minimal energy. At cycles of displacement amplitudes of $1.0 \Delta y, 2 \Delta y, 4 \Delta y, 6 \Delta y, 8 \Delta y, 10 \Delta y$, and $12 \Delta y$, the energy dissipated represents the sum of energy dissipation for three repetitive cycles. Figures 12 and 13 show the accumulated energy dissipation of square and circular braces for a particular width/diameter-to-thickness ratio and varying slenderness ratios. The value of dissipated energy consistently increases with number of cycles, and the square and circular braces with a slenderness ratio of 50 dissipated maximum energy at $\mathrm{B} / \mathrm{t}=8$ and $\mathrm{D} / \mathrm{t}=8$. For all width/diameter-to-thickness ratios, the ultimate magnitude of energy dissipation reduced with the increase in slenderness ratio. Thus, braces with smaller slenderness and width/diameter-to-thickness ratios have enhanced energy dissipation abilities. 

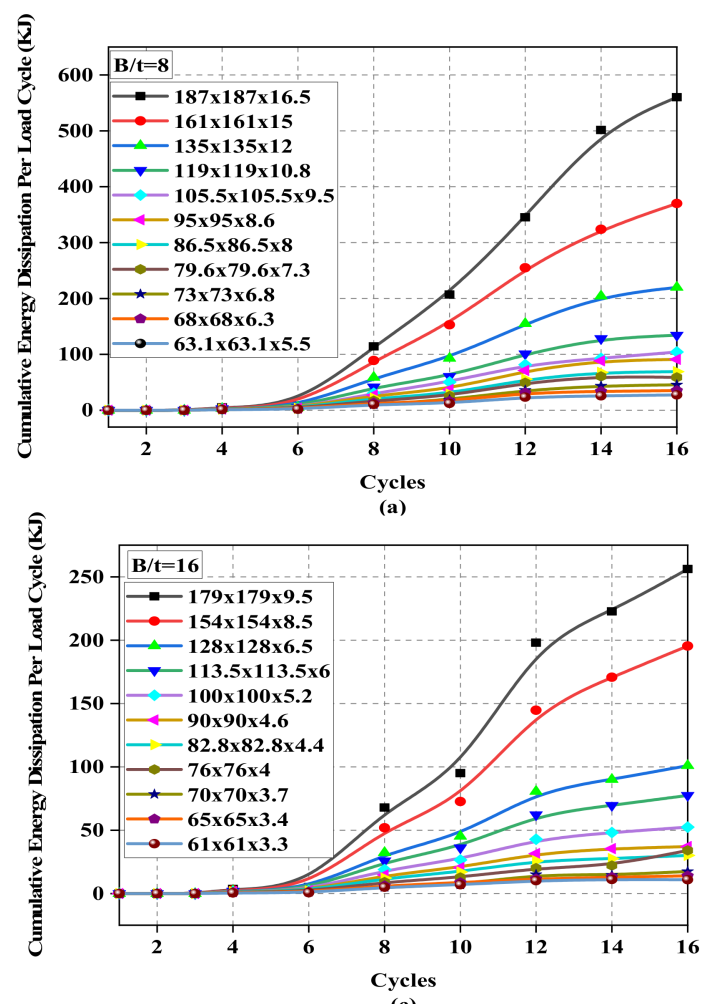

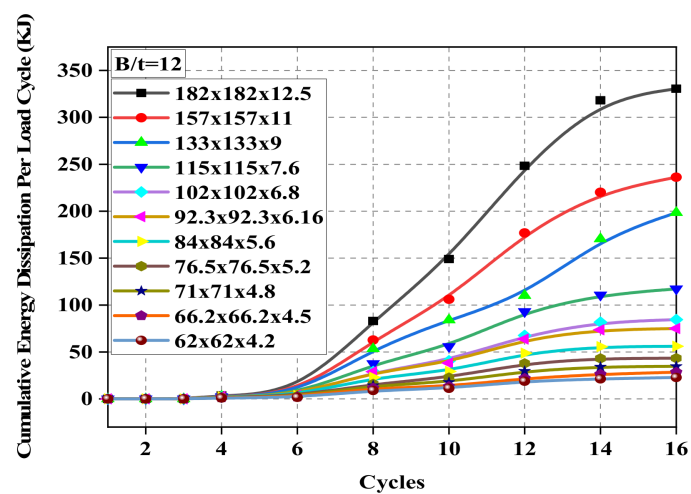

(b)

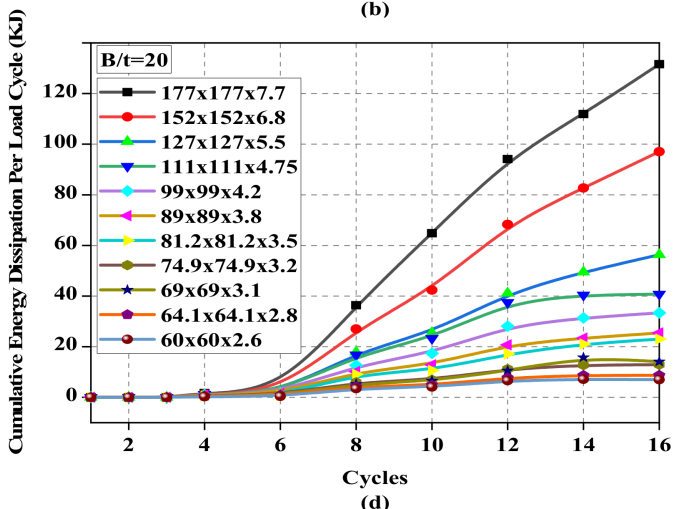

(d)

Figure 12. Cumulative energy dissipation of square braces for slenderness ratios $(\lambda=50-150)$ : (a) width-to-thickness ratio $=8$; (b) width-to-thickness ratio $=12$; (c) width-to-thickness ratio $=16$; (d) width-to-thickness ratio $=20$.
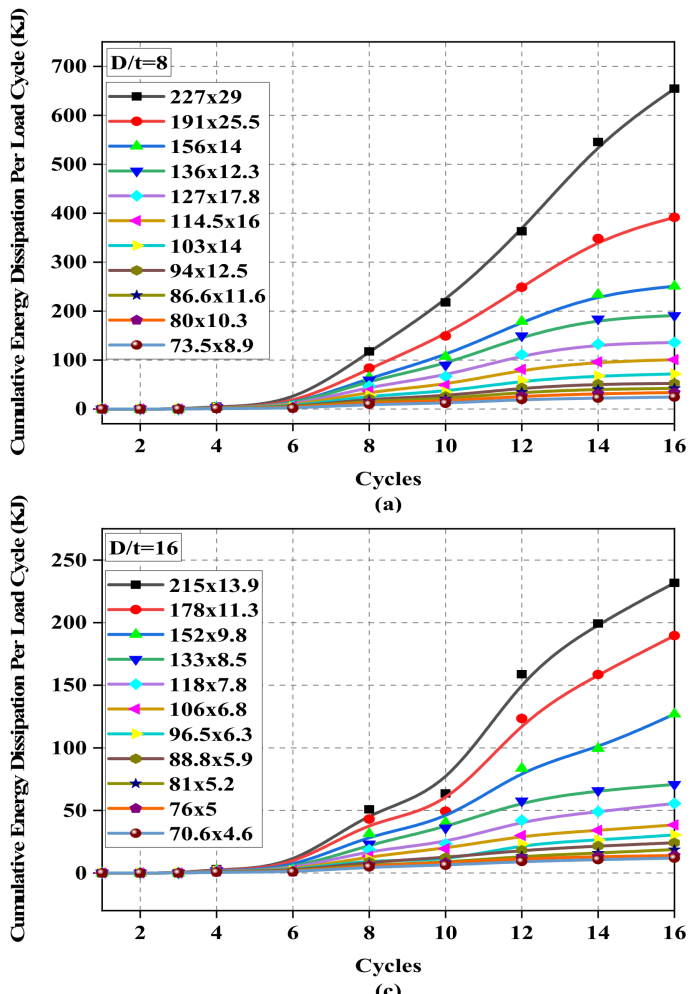
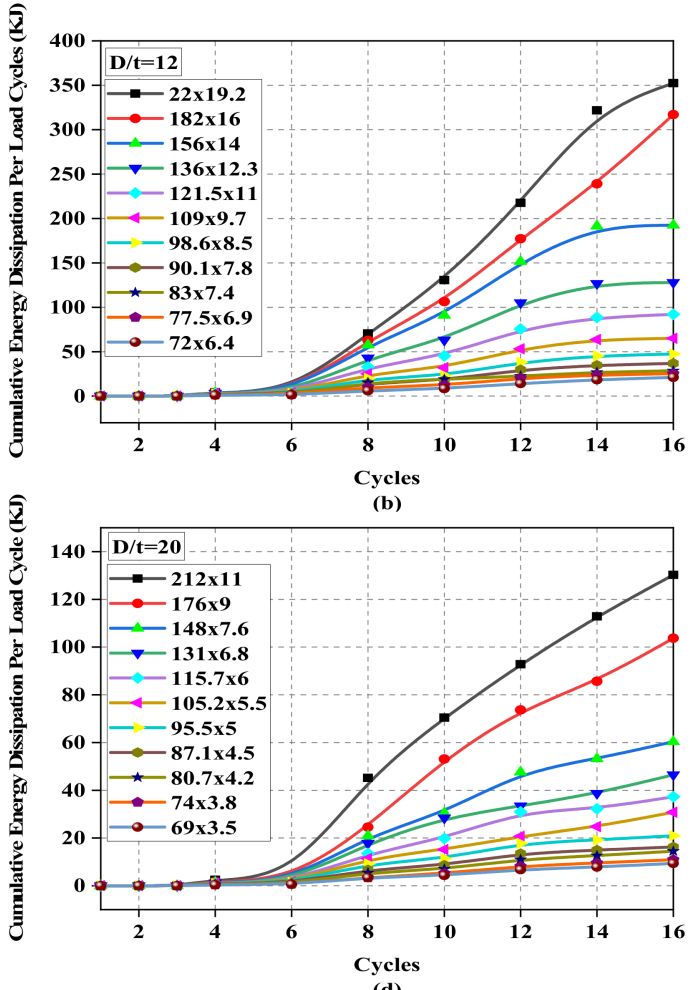

(d)

Figure 13. Cumulative energy dissipation of circular braces for slenderness ratios $(\lambda=50-150)$ : (a) diameter-to-thickness ratio $=8 ;(\mathbf{b})$ diameter-to-thickness ratio $=12 ;(\mathbf{c})$ diameter-to-thickness ratio $=16 ;(\mathbf{d})$ diameter-to-thickness ratio $=20$. 


\section{Discussion}

The numerical study performed in this article confirms the concept illustrated by previous investigations. The results showed the pattern in which a decrease in the energy dissipation capacity of steel braces under cyclic loading is connected with the increase in brace slenderness and width/diameter-to-thickness ratios. A large series of parametric experiments were undertaken to determine the effect of compressive-tensile resistance ratios, on a broad range of slenderness ratios and width/diameter ratios, of both square and circular shaped braces. This study provides a comprehensive outline of the collection of various slenderness ratios and width/diameter-to-thickness ratios that should be chosen for the use of these steel braces in concentric bracing frames.

As shown by the results, certain compressive resistance is retained after the braces undergo global buckling. The degraded compressive resistance is withstood through the establishment of plastic hinges at the middle and end sections of the braces, and the resistance steadily reduces with the loading cycles of large amplitudes. Tremblay [11] gathered the test results of various specimens with symmetrical and unsymmetrical loading protocols, and proposed the predictive curves for the compressive resistances after the occurrence of brace buckling. Figure 14 displays the normalized compressive resistances of the braces of the current FEA study, in conjunction with the predictive curves of Tremblay [11] and AISC [23], at loading displacements of $2 \Delta y$ and $4 \Delta y$ against a non-dimensional slenderness ratio $\bar{\lambda}$, which is defined as:

$$
\bar{\lambda}=\sqrt{A F_{y} / N_{c r}}
$$

where $A$ is the cross sectional of brace section, $F_{y}$ is the yield strength of the adopted material, and $N_{c r}$ is the elastic-critical buckling load. The compressive resistances of the modeled braces degrade with the increase in slenderness ratio and show good agreement with the predictive curve of Tremblay [11] at $2 \Delta y$, and have deviations at $4 \Delta y$. However, the AISC [23] prediction remained over-conservative in both cases.
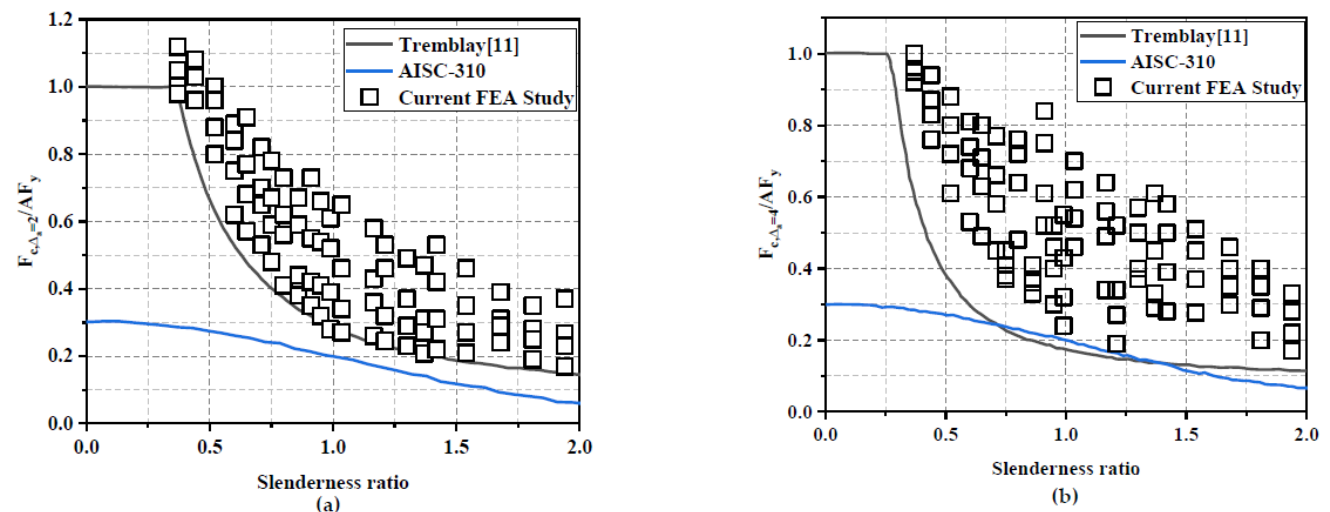

Figure 14. Comparison of normalized compressive resistances: (a) at loading displacement of $2 \Delta y$; (b) at loading displacement of $4 \Delta y$.

\section{Conclusions}

A comprehensive numerical study of hot-form carbon steel square and circular hollow braces under axial cyclic loading was carried out and presented in this paper. A series of 88 braces with varying width/diameter-to-thickness ratios and slenderness ratios were modeled to analyze cyclic behavior and compressive resistance degradation. The following conclusions can be drawn:

- The overall cyclic response of both square and circular braces was comparatively affected by diameter/width-to-thickness ratios and slenderness ratios.

- Both square and circular shaped hollow braces, with smaller slenderness and width/ diameter-to-thickness ratios, have better hysteretic performance. However, circularshaped braces generally showed a superior performance than square-shaped braces. 
- For all width/diameter-to-thickness ratios, the ultimate magnitude of energy dissipation reduced with the increase in the slenderness ratio. Thus, braces with smaller slenderness and width/diameter-to-thickness ratios have enhanced energy dissipation abilities.

- The compressive-tensile resistances significantly degraded with increasing cycles after the occurrence of the global buckling of the braces. The global buckling of slender specimens occurred earlier and compressive-tensile resistances were reduced more promptly compared to stocky members. Finally, the conclusion can be drawn that the width/diameter-to-thickness ratios have a paramount influence on the compressivetensile resistance degradation of steel braces under axial cyclic loading. By comparison, the slenderness ratio was found to be less significant.

Author Contributions: Conceptualization, H.H.; Data curation, A.S.; Funding acquisition, X.G.; Investigation, A.S.; Methodology, H.H.; Supervision, X.G.; Writing—original draft, H.H.; Writingreview \& editing, X.G. All authors have read and agreed to the published version of the manuscript.

Funding: This research received funding from the Beijing Natural Science Foundation Key Project, (8141001) and the National Natural Science Foundation Project of China, (51378038).

Institutional Review Board Statement: Not applicable.

Informed Consent Statement: Not applicable.

Data Availability Statement: Data available within the article.

Conflicts of Interest: The authors declare no conflict of interest.

\section{References}

1. Sheehan, T.; Chan, T.M. Response of hollow and concrete-filled CHS braces under cyclic loading. Structures and Buildings. Proc. Inst. Civ. Eng. 2014, 167, 140-152.

2. Gao, X.; Li, Y.; Li, J.; Xu, J. Effect of steel braces buckling on inelastic torsion and design prevention of steel braced concrete frame structure. In Structures; Elsevier: Amsterdam, The Netherlands, 2020; Volume 28, pp. 1687-1700. [CrossRef]

3. Sabelli, R. Research on Improving the Design and Analysis of Earthquake-Resistant Steel-Braced Frames; EERI: Oakland, CA, USA, 2001; $142 \mathrm{p}$.

4. Uriz, P. Toward Earthquake-Resistant Design of Concentrically Braced Steel-Frame Structures; Pacific Earthquake Engi-neering Research Center: Berkeley, CA, USA, 2008.

5. Roeder, C.W.; Lumpkin, E.J.; Lehman, D.E. A balanced design procedure for special concentrically braced frame connections. J. Constr. Steel Res. 2011, 67, 1760-1772. [CrossRef]

6. Palmer, K.D.; Roeder, C.W.; Lehman, D.E.; Okazaki, T.; Shield, C.K.; Powell, J. Concentric X-braced frames with HSS bracing. Int. J. Steel Struct. 2012, 12, 443-459. [CrossRef]

7. Yoo, J.H. Effect of Mid-span Gusset Plates on the Behavior of Multi-Story X-Braced Frames. J. Korean Soc. Steel Constr. 2013, 25, 179-186. [CrossRef]

8. Chen, C.H.; Lai, J.W.; Mahin, S. Seismic performance assessment of concentrically braced steel frame buildings. In Proceedings of the 14th World Conference on Earthquake Engineering, Beijing, China, 12-17 October 2008.

9. Sen, A.D.; Sloat, D.; Pan, L.; Roeder, C.W.; Lehman, D.E.; Berman, J.W. Evaluation of the seismic performance of two-story concentrically braced frames with weak beams. In Proceedings of the 5th International Conference on Advances in Experimental Structural Engineering, Taipei, Taiwan, 8-9 November 2013.

10. Costonzo, S.; D'Aniello, M.; Landolfo, R.; Martino, A.D. The influence of the beam flexural stiffness in the seismic response of inverted-V concentric braced frame. In Proceedings of the EUROSTEEL, Naples, Italy, 10-12 September 2014.

11. Tremblay, R. Inelastic seismic response of steel bracing members. J. Constr. Steel Res. 2002, 58, 665-701. [CrossRef]

12. Shaback, B.; Brown, T. Behaviour of square hollow structural steel braces with end connections under reversed cyclic axial loading. Can. J. Civ. Eng. 2003, 30, 745-753. [CrossRef]

13. Tremblay, R.; Haddad, M.; Martinez, G.; Richard, J.; Moffatt, K. Inelastic cyclic testing of large size steel bracing members. In Proceedings of the 14th World Conference on Earthquake Engineering, Beijing, China, 12-17 October 2008; pp. 12-17.

14. Roufegarinejad, A.; Tremblay, R. Finite element modeling of the inelastic cyclic response and fracture life of square tubular steel bracing members subjected to seismic inelastic loading. Behav. Steel Struct. Seism. Areas 2012, 97-103.

15. Nip, K.; Gardner, L.; Elghazouli, A. Cyclic testing and numerical modelling of carbon steel and stainless steel tubular bracing members. Eng. Struct. 2010, 32, 424-441. [CrossRef]

16. Kumar, P.A.; Sahoo, D.R. Fracture ductility of hollow circular and square steel braces under cyclic loading. Thin Walled Struct. 2018, 130, 347-361. [CrossRef]

17. ABAQUS/Standard Version 6.10, User's Manual; Hibbit, Karlsson and Sorensen Inc.: Providence, RI, USA, 2011. 
18. ECCS 1986. Recommended Testing Procedure for Assessing the Behaviour of Structural Steel Elements under Cyclic Loads; European Convention for Constructional Steelwork: Brussels, Belgium, 1986.

19. Chaboche, J. Time-independent constitutive theories for cyclic plasticity. Int. J. Plast. 1986, 2, 149-188. [CrossRef]

20. Nip, K.; Gardner, L.; Davies, C.; Elghazouli, A. Extremely low cycle fatigue tests on structural carbon steel and stainless steel. J. Constr. Steel Res. 2010, 66, 96-110. [CrossRef]

21. ANSI/AISC 341-16. Seismic Provisions for Structural Steel Buildings; American Institute of Steel Construction (AISC): Chicago, IL, USA, 2016.

22. Ministry of Housing and Urban-Rural Development of the People's Republic of China (MOHURD). Code for Seismic Design of Buildings; GB 50011-2010; Ministry of Housing and Urban-Rural Development of the People's Republic of China (MO-HURD): Beijing, China, 2010.

23. ANSI-A; AISC 341-10. Seismic Provisions for Structural Steel Buildings; American Institute of Steel Construction: Chicago, IL, USA, 2010 